\title{
EL SISTEMA EDUCATIVO EN EL 2000. OBJETIVOS MÚLTIPLES, EFICACIA ÚNICA
}

\section{THE EDUCATION SYSTEM IN 2000. MULTIPLE OBJECTIVES, ONLY EFFECTIVE}

\section{AUTORES}

Luis A. García Moreno: Catedrático y Miembro del Consejo de Universidades. luis.garcia@ozu.es

\section{CURRÍCULUM VITAE}

Profesor en la Facultad de Filosofía y Letras de la Universidad de Alcalá de Henares (Departamento de Historia I y Filosofía).

\section{RESUMEN}

A fines del siglo XX es indudable que un sistema educativo avanzado, como debe ser el español, no puede ni debe cumplir con un sólo objetivo. De hecho en ninguna parte y en tiempo alguno ha sido diseñado para una sola finalidad. Sin duda, algunos pudieran pensar que tal afirmación no deja de ser una perogrullada. Sin embargo, como tantas otras cosas en las Ciencias del Hombre no se ha tenido en cuenta a la hora de realizar muchos análisis de la realidad presente y a la hora de arbitrar soluciones de futuro. Y ello, en el caso que nos ocupa de la Educación, porque frecuentemente una mayoría de los destinatarios de la misma no consideran que deba cumplir más de un objetivo; o porque según los tiempos ha primado especialmente una finalidad, cuando menos en cada uno de los diversos niveles en que se ha podido articular el mismo. 


\section{PALABRAS CLAVE}

Sistema educativo - Objetivos multiples - Eficacia única

\section{ABSTRACT}

In the late twentieth century is no doubt that an advanced education system, as it must Spanish, can not and must meet a single objective. In fact anywhere and any time is designed for one purpose. Undoubtedly, some might think that such a claim no longer a truism. However, like many other things in the Sciences of Man has not been taken into account when making a lot of analysis of present reality and time to arbitrate future solutions. And this, in the present case of education, because often a majority of recipients do not consider it to be met by more than one objective, or because as time has prevailed especially a purpose, at least in each of the different levels has been able to articulate it.

\section{KEY WORDS}

Education system - Multiple Objectives - Effective only

\section{ÍNDICE}

1. Preámbulo

2. Objetivos educativos a lo largo de la Historia

3. Escuela comprehensiva e igualitarismo (EEUU, Japón y España)

4. La Escuela alemana: la funcionalidad de la diversidad

5. Convergencia de Sistemas : Alemania y Japón

6. Una posible vía educativa para España 


\section{Preambulo}

A fines del siglo XX es indudable que un sistema educativo avanzado, como debe ser el español, no puede ni debe cumplir con un sólo objetivo. De hecho en ninguna parte $\mathrm{y}$ en tiempo alguno ha sido diseñado para una sola finalidad. Sin duda, algunos pudieran pensar que tal afirmación no deja de ser una perogrullada. Sin embargo, como tantas otras cosas en las Ciencias del Hombre no se ha tenido en cuenta a la hora de realizar muchos análisis de la realidad presente y a la hora de arbitrar soluciones de futuro. Y ello, en el caso que nos ocupa de la Educación, porque frecuentemente una mayoría de los destinatarios de la misma no consideran que deba cumplir más de un objetivo; o porque según los tiempos ha primado especialmente una finalidad, cuando menos en cada uno de los diversos niveles en que se ha podido articular el mismo.

\section{Objetivos educativos a lo largo de la Historia}

Simplificando al máximo se puede decir que a lo largo de la Historia educativa de Occidente tres han sido los objetivos básicos que se ha pretendido que cumpliera la Educación : (1) enseñar un conjunto de conocimientos y habilidades básicos para desenvolverse con soltura en la vida, o específicos para desarrollar una determinada actividad profesional o para reproducir unas determinadas elites ; (2) dotar de unos presupuestos ideológicos, de un ideario e imaginario colectivos, que garanticen una máxima homogeneidad cultural de ideológica y una apariencia, más o menos real, de igualdad social y/o de oportunidades ; (3) articular los diversos segmentos sociales en un máximo de armonía, que evite los peligros para el conjunto de la marginación de algunas minorías, socializando tanto los mismos procedimientos del aprendizaje de conocimientos y habilidades como el mismo ocio, al menos en las etapas infantiles y juveniles. 
Un atento escrutinio de la evolución de los sistemas educativos en nuestra Civilización occidental -desde la Grecia clásica a nuestros días- nos llevaría a descubrir que los tres objetivos han estado prácticamente presentes en todos ellos y en los diversos niveles de enseñanza, desde las escuelas de primeras letras a los estudios universitarios, incluso de postgrado. Aunque también resulta evidente que cada una de esas tres series de objetivos han primado, y deben primar, de manera muy diferente según los tiempos y las sucesivas etapas educativas.

Intuitivamente se podría pensar que el primer objetivo fue prioritario en el mismo nacimiento de los procesos educativos reglados y socializados en Occidente. Sin embargo una atenta mirada, por ejemplo, al famoso sistema educativo de la Esparta clásica, que tanta admiración ha causado entre sus contemporáneos pero también en tiempos muy posteriores, no muestra que las series de objetivos antes mencionados en segundo y tercer lugar tenían tanta importancia como los primeros, en definitiva centrados en la lucha y tácticas propias del guerrero hoplita. Por el contrario, no se puede negar que el primer conjunto de objetivos fue central en el surgimiento y desarrollo de las instituciones universitarias medievales.

No obstante sería equivocado pensar que en la Universidad europea tradicional hubo completa uniformidad a la hora de jerarquizar los objetivos a alcanzar a través de su quehacer. Pues por un lado se puede hablar del modelo boloñés, ciertamente el más extendido, destinado a producir profesionales, especialmente juristas, necesitados por la creciente complejidad de los Estados y sociedades medievales ; y de ahí vino la organización de la corporación universitaria, y la misma jerarquización de los grados académicos, a imitación de las propias de los gremios artesanales. Pero junto a éste muy pronto surgió el modelo parisino ideado por Roberto de Sorbon, en el que la pura especulación, esencialmente filosófica y teológica, primaba de manera diáfana sobre la producción de especialistas y profesionales. Indudablemente ambos tipos de instituciones universitarias -que en el caso español muy bien se podrían personalizar, 
para principios del siglo XVI, en Salamanca y Alcalá, respectivamente- tenían una consecuencia ineludible : la reproducción de una elite social con un determinado perfil cultural e ideológico. Por ello que en España, por ejemplo, los grados universitarios llevaran aparejados en el Antiguo Régimen los privilegios de nobleza, aunque no hereditarios, y que los egresados de alguna Universidad, como Salamanca, jurasen defender determinados Dogmas del Catolicismo. En fin, basta leer las vivencias universitarias de nuestros escritores del Siglo de Oro para comprender también que, aunque de una manera secundaria, también esas instituciones educativas sirvieran a algunos de los objetivos seriados anteriormente en segundo y tercer lugar : desde la integración de elementos marginales de la sociedad hasta facilitar una cierta labilidad social, al constituir una de las pocas instancias de la época donde existía una teórica igualdad de oportunidades entre personas de condición social y económica muy diferentes.

En España, como en el resto de Occidente, el surgimiento en el siglo XIX de una primera enseñanza universal y obligatoria fue fruto del Liberalismo y del Estado nacional moderno. En definitiva, el establecimiento de una Escuela universal y laica se constituyó en símbolo principalísimo de la sustitución de la Iglesia por el Estado como guardián y propagador de del conjunto de valores y creencias en que debía sustentarse el nuevo Estado nacional. Desde este punto de vista no cabe duda que la antes señalada segunda serie de objetivos de todo sistema educativo pasó a un plano principal, cuando menos en los primeros grados del nuevo sistema educativo. Además este último en la mayor parte de Europa, y también en España, se configuró como estatal o, cuando menos, bajo la estrecha supervisión del Estado, llegando incluso a convertir a los maestros y profesores en funcionarios públicos. Por ello no puede extrañar que la misma eficacia del sistema, como instrumento de transmisión de conocimientos positivos, se sacrificara no sólo a esos objetivos de homogeneización ideológica, sino también a la máxima igualdad en los conocimientos y habilidades de toda la población estudiantil de una misma edad. Sin 
duda que el desideratum de esta tendencia sería el surgimiento de la llamada Escuela comprehensiva, especialmente impulsada en toda Europa por los partidos socialistas.

Ese objetivo igualitarista, y su corolario de pérdida de eficacia en el aprendizaje de habilidades y conocimientos específicos, en los sistemas educativos de la Europa del siglo XX tuvo su piedra de toque en la Secundaria. Como es sabido fue éste el escalón que más tardíamente se configuró en los sistemas educativos occidentales. En España, como en buena parte de la Europa continental, la Secundaria se inspiró en el modelo francés, surgiendo formalmente como un producto de la radical reforma que de las Universidades realizó el nuevo Estado liberal, heredando las funciones preparatorias para estudios especializados y profesionales superiores que habían sido propios de las llamadas Facultades Menores, básicamente la antigua de Artes. Como consecuencia lógica de ello los Institutos españoles -como sus equivalentes los Liceos franceses, portugueses e italianos, o los Gimnasios de los países de cultura alemana- se constituyeron como esenciales instrumentos educativos preparatorios para la entrada en la Universidad, en los que primaban los elementos académicos y la consiguiente transmisión de saberes, así como de habilidades y valores propios de una elite cultural y social.

\section{Escuela comprehensiva e igualitarismo (EEUU, Japón y España)}

Por razones históricas el sistema educativo de los Estados Unidos de América tuvo desde el principio elementos híbridos de los que se desarrollaron en el Continente europeo, tras la Revolución francesa, y de la propia tradición inglesa, más conservadora y respetuosa con las particulares creencias religiosas. Además de ello el sistema educativo americano tuvo que vérselas con el brutal crecimiento demográfico de la nación en el siglo XIX y primeros decenios del XX, principalmente debido a la venida de emigrantes de lengua y tradiciones nacionales diversas. Por último, la Educación en los EEUU se vio con frecuencia sometida a la tentación 
utilitarista, siempre presente en la llamada mentalidad yanki. Todo ello desembocó en un sistema educativo polimórfico, en el que los objetivos a perseguir, y su plural institucionalización, variaban según sus diversas etapas o grados. Como característicamente americano podríamos citar el especial interés del Estado por asegurar una enseñanza secundaria lo más uniforme y universal posible, lo que se institucionalizó en las High Schools de titularidad pública. La etapa secundaria sin duda se convirtió así un el más eficaz instrumento de la política del llamado Melting pot, o mixturación étnica. Por tanto, los objetivos señalados en la segunda de las series enunciadas al principio, igualitarismo y homogeneización en primer lugar, han sido y siguen siendo prioritarios en la etapa secundaria del sistema educativo americano. Bastante antes que en otros países occidentales la High School americana asumió mucho elementos propios del ideal de comprehensividad, al tiempo que incluía en sus curricula bastantes actividades que vistas desde Europa parecían enormemente triviales, como era el gran lugar concedido a la práctica del deporte y juegos asociativos, a las llamadas enseñanzas "domésticas" y a otra serie de actividades para-académicas en las que primaba el factor asociativo y socializador del grupo escolar. Es curioso que este tipo de escuela ya en los años treinta del presente siglo levantara las críticas más feroces de un gran teórico y práctico de la reforma educativa en las Universidades americanas como fue Abraham Flexner. Pues posiblemente con razón el padre del "Instituto de Estudios Avanzados" de Princeton veía en la elevada comprehensividad y triviliadad curricular de la High School especialmente comparada con el Liceo francés o el Gimnasio alemán- la razón básica de la mediocridad de la Universidad americana de la época, frente a las europeas, especialmente la alemana que Flexner admiraba. Por su parte la incidencia del utilitarismo americano se hacía especialmente notar en los estudios universitarios y profesionales (Vocationals en la jerga americana). Desde la falta de regulación estatal de muchas enseñanzas profesionales -incluida la Medicina hasta la gran reforma impulsada por el propio Flexner ya en el siglo XX-, hasta el nacimiento de esa institución tan típicamente de los Estados del corn belt como fueron las 
"Universidades agrícolas", hasta la mucho más reciente y prometedora de las Universidades de reciclaje profesional.

Durante muchos años, así pues, la High School fue la gran paleta mezcladora del Melting Pot americano para los jóvenes blancos. Las conocidas leyes integradoras impulsadas por la New frontier kennedyana la hizo extensible también para los negros y otras minorías étnicas, pero ya en un marco radicalmente cambiado, puesto que el objetivo del "mortero fundidor" se veía sustituido por el del respecto a la diversidad étnica y el reconocimiento, como lo único "políticamente corrector", de los EEUU de América como una nación pluriétnica y pluricultural. A partir de entonces no cabe duda que los objetivos señalados al principio en tercer lugar -la cohesión social, la lucha contra la marginación social o cultural de las minorías, la socialización del ocio de todos los adolescentes incluso mediante su misma institucionalización en el proceso educativo- han venido teniendo un protagonismo espectacular en la Secundaria americana. En este contexto lógicamente que las razonables críticas que hace más de medio siglo dedicó a la High School de su patria han dejado de tener una parte de su sentido. Pues Flexner criticaba la endeblez académica de una porción del curriculum escolar, y su sustitución por otras actividades más triviales y de ocio, así como su escaso carácter selectivo y benevolencia evaluadora, con la consecución de la graduación por la inmensa mayoría de los estudiantes. Pues precisamente todo ellos son objetivos perseguidos hoy, incluso por ley. Es más, ambos objetivos en un cierto grado se han trasladado hoy al periodo de graduación (Bachelor of Arts/Science) del sistema universitario americano, que sociológicamente ha venido a representar el papel que tenía antes de la II Guerra Mundial la High School. Basta a este respecto pensar en la moderna legislación americana sobre la discriminación positiva a la hora del ingreso en la Universidad y del avance en la misma para determinadas minorías étnicas o sexuales. 
Es sabido que uno de los objetivos principales, y declarado, de la ocupación americana del Japón tras su derrota en 1945 fue la erradicación de las élites culturales y sociales del periodo Meiji que habían sostenido el denostado imperialismo y etnocentrismo nipón, así como su reproducción. Instrumento para lograrlo fueron una Constitución democrática y parlamentarista, la confiscación de las grandes corporaciones financieras e industriales vinculadas a unas cuantas familias relacionadas con la dinastía imperial, y una reforma radical del sistema educativo, aniquilando tanto el sesgo elitista como la estricta vinculación del estudio con el servicio al Estado con la derogación del famoso Decreto Imperial sobre Educación de 1890. En su lugar se impuso un sistema uniforme, universal y lo más comprehensivo posible desde los 6 a los 15 años, con una Secundaria Superior casi de las mismas características. Se conformó así el conocido sistema actualmente vigente de 6+3+3 años, uniforme y jerarquizado, en la que la única diversidad se establece en el último peldaño, con la concurrencia, claramente minoritaria, de los Colegios técnicos, con una enseñanza en parte compatible ya con el trabajo.

El sistema educativo japonés, impuesto por los EEUU en gran parte a imitación del suyo, solamente está sometido en teoría a un único filtro, exactamente como el americano : la entrada en la Universidad. Acceso que es muy restrictivo si el alumno pretende estudiar en alguna de las Universidades públicas de prestigio. Cualquier persona familiarizada por el sistema educativo español podrá así observar que éste es muy parecido al de uno y otro.

Como en otros países europeos desde mediados del siglo XIX, con la famosa Ley Moyano, se instauró en España un sistema educativo horizontal, en el que coexistían diversas rutas para alumnos de una misma edad. La más prestigiosa de ellas era el Bachillerato, muy tutelado y vigilado por el Estado, que tenía por fin dar una formación muy académica con la idea de preparar a una elite para los posteriores estudios universitarios. El Bachillerato español clásico se iniciaba ya con una fuerte 
selectividad, el famoso "ingreso", con ejercicios orales y escritos ante un tribunal. Puede decirse que hasta los años cincuenta todas las reformas que se hicieron en ese Bachillerato fueron para endurecerlo y hacerlo más selectivo bajo la estricta vigilancia del Estado por medio de sus profesores-funcionarios, y reforzar la estrecha ligazón de sus últimos años con la Universidad : desde el Examen de Estado a la Prueba del Preuniversitario, pasando por las Revalidas elemental y superior. Junto a esta Secundaria selectiva y muy académica se fueron desarrollando otras enseñanzas para ese tracto de edad, con un carácter más profesional y menos selectivo, y desde luego prestigiado socialmente : desde las antiguas Escuelas de Artes y Oficios hasta las Universidades Laborales del Franquismo. La famosa Ley General de Educación de 1970 supuso un cambio radical en tan antañón edificio, introduciendo de una manera decisiva en el sistema educativo español la idea de la escuela comprehensiva, cuyos pilares básicos eran la nueva Educación General Básica y el nuevo Bachillerato Unificado y Polivalente. La reforma llegaba a España más tarde que a otros países y, como casi siempre en esos casos, con mayor radicalidad. Esta última se exacerbó finalmente con la instauración, ya en los ochenta, de la Ley de Organización General del Sistema Educativo (LOGSE). Esta última culminaba el camino hacia la comprehensividad total, guiada por el dogmatismo del PSOE, ya claramente trasnochado en asuntos de Educación. No obstante que algunos expertos del propio Gobierno del PSOE -como se documenta en el Libro Blanco que precedió a la leyabogaban por establecer algún control al final del último tramo de la Secundaria, lo cierto es que por claras motivaciones populistas finalmente se optó por dejar como único filtro, a la vez mixturado de validación de los estudios previos, la ya vieja prueba de la Selectividad, a realizar por aquellos alumnos que fueran a proseguir sus estudios en la Universidad.

\section{La Escuela alemana : la funcionalidad de la diversidad}


Sin duda que la tendencia hacia la comprehensividad en la escuela, y más concretamente en la etapa de la Secundaria, ha sido general en todo el Occidente tras la II Guerra Mundial, por las razones que se señalaron al principio. Es más, los años setenta incluso recibieron un último empujón, de la mano específicamente de partidos socialdemócratas, que convirtieron así a la Educación en un caballo de batalla fundamental de la lucha política, lo que nunca puede ser positivo. Especialemnte el proceso se documenta muy bien en Alemania. La República Federal, sorprendentemente al contrario de lo ocurrido en Japón, fue capaz de continuar con su tradicional y tripartito sistema escolar heredado del Reich, no obstante ciertos esfuerzos norteamericanos por introducir otro comprehensivo. Dicho sistema se basa en la clasificación de los jovencitos desde muy pronto, al principio de la Secundaria, en tres tipos de escuelas : Hauptschule, Realschule y Gymnasium. Tripartición que refleja una clara jerarquización tanto en prestigio social como en capacidades de los alumnos, así como una diversidad de opciones profesionales. En la base, la Hauptschule, con una única salida a enseñanzas profesionales poco cualificadas y ya en un sistema dual, cen el que los estudios son compatibles con un trabajo de aprendiz. La Realschule contiene ya una cierta dosis de selectividad a su entrada, y capacitará para proseguir posteriormente enseñanzas técnicoprofesionales medias y superiores (Fachoberschule y Fachochschule). En la cúspide del prestigio y la selectividad el clásico Gymnasium, dividido en dos fases, entre sí selectivas, y paralela la superior a los estudios técnicos profesioanles antes mencioandos, al menos en sus primeras etapas. Los estudios del Gymnasium culminan con unas complejas pruebas evaluadoras, cuya superación (Abitur) permite la entrada, sin mayores restricciones ni selectividad, en la Universidad.

En los años sesenta la comprehensividad se intentó introducir mediante la creación de una nueva escuela unitaria, la Gesamtschule. Ello se convirtió en un objetivo propio de los gobiernos socialdemócratas que trataron de imponerlo en los Länder que mejor controlaban, como Hesse o Rin Septentrional-Westfalia-. Sin embargo el 
intento chocó frontalmente con la mayoría de las organizaciones de padres de alumnos y con el partido Cristiano-Demócrata, y muy especialmente con su ala bávara, los Social-Cristianos, de modo que los Lánder siempre controlados por éstos, como Baviera y Baden-Wüttenberg, se convirtieron auténtico coto cerrado al invento. Esa oposición frontal a la escuela comprehensiva, tratando de demostrar con estadísticas en mano su poca eficiencia, su carácter caótico y su auténtica escasa capacidad de lograr la igualdad de oportunidades entre los estudiantes provenientes de distintas clases sociales, se plasmó muy especialmente en el famoso manifiesto del 1989 hecho por una mayoría de especialistas en Educación, mediando con la ley de 1986 de "Libertad escolar" hecha por los Cristiano-Demócratas al conseguir por vez primera el poder en Hesse. La incorporación de los Länder de la antigua República Democrática tampoco fue capaz de desequilibrar la batalla, sino más bien lo contrario.

\section{Convergencia de Sistemas : Alemania y Japón}

Sin embargo no podría decirse que la batalla, no obstante la aparente victoria de la contrarreforma, haya dejado incambiado el tradicional edificio tripartito de la Escuela alemana. El aumento espectacular de la demanda de Educación superior en Alemania, como en el resto de los países europeos, a partir de la década de los sesenta ya de por sí obligaba a aumentar la capacidad de las escuelas que conducían a la Universidad, que necesariamente tenían que ser los Gimnasios si no se querían crear las problemáticas Gesamtschulen. Y precisamente eso fue lo que se hizo, especialmente en aquellos Länder donde más poder ha tenido la CDU. Paralelamente se procedió a quitar rigidecesl proceso selectivo en la Secundaria, sustituyendo la prueba intermedia, para pasar a la segunda etapa del Gymnasium, por un periodo de observación y evaluación continua del alumno a lo largo de uno o dos años. Además se procuró aminorar la misma dureza de esta última, de modo que entre 1990 y 1991 se pasó de una tasa del $48 \%$ de los alumnos del Gimnasio que lograban su Abitur, a 
otra del 76 \%. Mientras que por otra parte se apoyó a la Realschule en detrimento de la antigua y desprestigiada Hauptschule. Para ello se crearon pasarelas que permitían el paso de éstas, incluso ya en el sistema dual, a los Gimnasios, y desde las enseñanzas técnicas a la Universidad. Al tiempo que las enseñanzas superiores de las Fachochschulen se hacían equivalentes en su consideración legal a la primera fase de los estudios universitarios; lo que llevó aparejado, entre otras cosas, la equiparación de las recién creadas Gesamtuniversitäten, en principio muy técnicas y profesionales, a las Universidades tradicionales. De esta forma la proporción de la distribución de los estudiantes de Secundaria entre los tres tripos de escuelas había cambiado radicalmente a principios de esta década respecto a tiempos anteriores, acercándose a un tercio del total cada una y sobresaliendo el Gymnasium con la proporción más elevada, el 31,6\%.

Con estos cambios el sistema educativo alemán, caracterizado por ser uno de los menos comprehensivos de Occidente, ha limado sus aristas, consiguiendo un elevado grado de aceptación por parte de la misma sociedad. De tal modo que la elección de un tipo u otro de escuela va teniendo cada vez más su motivación en los diversos objetivos futuros que de la educación se trace cada grupo social. Así se observa cómo las familias de la clase trabajadora, de empleados autónomos y de tipo medio o "cuello blanco", muestran una tendencia a aceptar las recomendaciones de los profesores para que su hijos opten por la Realschul, aviniéndose así a no seguir estudios posteriores en la Universidad. Mientras que son las familias de funcionarios -los tradicionales Beamten alemanes- los que más se obstinan, por cuestión de tradición, en que sus hijos accedan a ésta.

Por su parte el sistema educativo japonés, uno de los menos jerarquizados y más próximo al ideal radical de la Escuela comprehensiva en todo Occidente, en las últimas décadas ha acabado por crear un propio sistema de jerarquización y producción de elites. Y ello se ha conseguido mediante el surgimiento de una 
durísima competitividad entre los diversos centros de Secundaria por hacerse con los alumnos intelectualmente mejor dotados y más esforzados. El método para ello empleado no ha sido otro que posibilitar la elección de centro por los alumnos, no forzando a los de familias de un determinado barrio a tener que ir necesariamente al mismo, mediante el establecimiento de varios en el mismo, que se convirtieron de inmediato en competidores a través de pruebas selectivas de ingreso. El Estado, por su parte, ha facilitado y provocado esa competitividad al publicar un exhaustivo listado de todas las escuelas, públicas y privadas, del país jerarquizadas según el éxito alcanzado en la preparación de sus alumnos para superar la selección para ingresar en la segunda etapa de la Secundaria en los centros más prestigiosos y, sobre todo, para acceder a las muy selectivas grandes Universidades públicas, también clasificadas junto a las múltiples privadas en razón de su mayor carácter selectivo. Una vez más el caso japonés viene a mostrar cómo en un país muy desarrollado social y económicamente la elección de una determinada enseñanza por parte de las familias de los alumnos viene determinada especialmente por los diversos objetivos que cada una de éstas pidan que tenga el sistema educativo. Así, en el que se ha venido en llamar "Capitalismo Confuciano", con un elevadísimo aprecio tradicional por la Educación letrada y formal, muchas familias trabajadoras estén dispuestas a hacer un esfuerzo adicional para que sus hijos accedan a los centros situados en una posición superior en ese ranking. Para ello no dudan en pagar sumas elevadas, superiores en muchos casos al medio millón de pesetas, a las famosas academias de fin de semana (jukus) que dan una enseñanza suplementaria para preparar esos exámenes de ingreso. De tal modo que actualmente la Escuela japonesa más que reproducir en parte las distinciones de clase, como en el resto de Occidente, de hecho las está creando por su propia cuenta.

Los ejemplos japonés y alemán, en su tendencia a la convergencia de resultados a partir de presupuestos diametralmente distintos, vendrían a demostrar cómo en los actuales sistemas educativos occidentales el reto de la eficiencia, la capacidad de 
formar elites, intelectuales y profesionales a la vez que socializar su servicio, se deben no tanto al diseño formal del mismo como a la correcta adjudicación de objetivos y su consecución en cada una de las sucesivas fases del proceso educativo. Conclusión que creemos que puede tener una gran importancia a la hora de reflexionar sobre el deseable perfeccionamiento del actual sistema educativo español.

\section{Una posible vía educativa para España}

Como dijimos anteriormente en España la LOGSE ha impuesto una escuela de marcada comprehensividad, hasta la ESO inclusive. Con independencia del juego de mayorías parlamentarias, y las posibilidades de un cambio radical de dicha ley, sinceramente creemos que lo más realista y útil puede ser tratar de hacer al sistema más eficaz. De este modo, en primer lugar, se puede pensar que en las etapas comprehensivas de nuestra Escuela -Primaria y ESO- no interesa tanto un debate interminable sobre sus contenidos positivos y la posibilidad de mejorarlos -en realidad algo muy difícil si no se quiere aumentar el fracaso escolar-, como priorizar a esas etapas los objetivos señalados en segundo y tercer lugar, muy especialmente estos últimos. Pues estos últimos parecen cada vez más necesitados de su desarrollo en el sistema educativo ante la menor operatividad, por razones que no son del caso comentar aquí, de sus tradicionales canales de difusión, como eran, especialmente, la familia, las comunidades rurales y la Iglesia ; mientras que, por el contrario, están aumentando los in puts negativos y contrarios a esos valores y objetivos, tanto desde la misma sociedad cada vez más inorgánica de individualista como desde poderosos mass media de alcance incluso planetario. Y en segundo lugar urge fomentar la capacidad de encauzamiento selectivo del paso a la Secundaria no obligatoria, especialmente el nuevo Bachillerato. Para ello, posiblemente, no parece fórmula desaconsejable crear una correcta y transparente competencia entre los diversos centros dedicados a esa etapa educativa para captar y preparar en las mejores condiciones a sus alumnos. 